cross sections would have been maximum at an energy of several kev. Since these reactions are symmetrical in character, it follows that either the neutralized ions or the newly-created ions are excited to proper energy levels or they are dissociated and excited so that the energy defect becomes equal to zero. The neutralized argon atom cannot be excited since its first excited state has 11.5-ev energy which is very large compared to the energy defects of these reactions. It thus becomes apparent that the ions formed by the process of transfer of charge viz., $\mathrm{CO}^{+}, \mathrm{NO}^{+}$, and $\mathrm{CO}_{2}{ }^{+}$are either excited or dissociated. If we assume that $\mathrm{D}\left(\mathrm{CO}^{+}\right)$is $8.43 \mathrm{ev}$, then it follows that the newly-created $\mathrm{CO}^{+}$ions in the reaction $\left(\mathrm{A}^{+}, \mathrm{CO}\right)$ cannot be dissociated because the energy defect $(\Delta E=1.76 \mathrm{ev})$ is small compared to the dissociation potential.

Owing to lack of data for $\mathrm{D}\left(\mathrm{CO}_{2}{ }^{+}\right)$, the reaction $\left(\mathrm{A}^{+}, \mathrm{CO}_{2}\right)$ cannot be analyzed. However, due to the small energy defect $(\Delta E=2.06 \mathrm{ev})$, it is expected that $\mathrm{CO}_{2}{ }^{+}$ions formed by charge transfer are excited. Since the energy defect $(\Delta E=6.5 \mathrm{ev})$ is large for the reaction $\left(\mathrm{A}^{+}, \mathrm{NO}\right)$, the resultant $\mathrm{NO}^{+}$ion may be dissociated. However, it is difficult to predict this result until $\mathrm{D}\left(\mathrm{NO}^{+}\right)$is known.

Like other reactions having negative energy defect, ${ }^{3}$ the reverse reactions $\left(\mathrm{CO}_{2}^{+}, \mathrm{A}\right)$ and $\left(\mathrm{CO}^{+}, \mathrm{A}\right)$ show a steady rise of cross section with ion energy indicating that energy balance has not been obtained in the observed energy range of 100 to $800 \mathrm{ev}$.

${ }^{1}$ S. N. Ghosh and W. F. Sheridan, J. Chem. Phys. 26, 480 (1957).

${ }^{2}$ Dillon, Sheridan, Edwards, and Ghosh, J. Chem. Phys. 23, 776 (1955).

${ }^{3}$ S. N. Ghosh and W. F. Sheridan, J. Chem. Phys. 25, 1076 (1956).

\section{Infrared Dichroism of Mutually Perpen- dicular Normal Modes in Oriented High Polymers}

C. Y. Liang* and S. Krimm

The Harrison M. Randall Laboratory of Physics, University of Michigan, Ann Arbor, Michigan

(Received June 12, 1957)

$\mathbf{I}^{\mathrm{T}}$ $T$ is known that associated with the normal modes of some units in a high polymer chain are three mutually perpendicular transition moments, as for example the $\nu_{s}\left(\mathrm{CH}_{2}\right), \nu_{a}\left(\mathrm{CH}_{2}\right)$, and $\gamma_{w}\left(\mathrm{CH}_{2}\right)$ modes of polyethylene, ${ }^{1}$ and the $\nu(\mathrm{OH}), \delta(\mathrm{OH})$, and $\gamma_{w}(\mathrm{OH})$ modes of polyvinyl alcohol. ${ }^{2}$ The purpose of this note is to examine the infrared dichroism of such modes in uniaxially oriented polymers, with particular reference to its structural implications.

For perfect uniaxial orientation, the dichroic ratio, $R$, defined as the ratio of optical densities with electric vector respectively parallel and perpendicular to the
TABLE I. Dichroism of mutually perpendicular normal modes.

\begin{tabular}{|c|c|c|c|c|c|c|}
\hline & $\phi_{1}$ & $\mathbf{M}_{1}$ & $\phi_{2}$ & $\mathbf{M}_{2}$ & $\phi 8$ & $\mathbf{M}_{3}$ \\
\hline 1. & $<\theta_{1}$ & $\pi$ & $>\theta_{2}$ & $\sigma$ & $>\theta_{2}$ & $\sigma$ \\
\hline 2. & $=\theta_{1}$ & $\pi$ & $\begin{array}{l}>\theta_{2} \\
=\theta_{2}\end{array}$ & $\begin{array}{l}\sigma \\
u\end{array}$ & $\geq \theta_{2}$ & $\underset{\sigma}{\sigma}$ or $u$ \\
\hline 3. & $\theta_{1}<\phi_{1}<\theta_{2}$ & $\pi$ & $\begin{array}{c}>\theta_{2} \\
=\theta_{2} \\
\theta_{1}<\phi_{2}<\theta_{2}\end{array}$ & $\begin{array}{l}\sigma \\
u \\
\pi\end{array}$ & $\begin{array}{l}>\theta_{1} \\
>\theta_{2} \\
>\theta_{2}\end{array}$ & $\begin{array}{c}\pi, u, \text { or } \sigma \\
\sigma \\
\sigma\end{array}$ \\
\hline 4. & $=\theta_{2}$ & $u$ & $\begin{array}{c}>\theta_{2} \\
=\theta_{2} \\
\theta_{1} \leq \phi_{2}<\theta_{2}\end{array}$ & $\begin{array}{l}\sigma \\
u \\
\pi\end{array}$ & $\begin{array}{l}<\theta_{2} \\
=\theta_{2} \\
>\theta_{2}\end{array}$ & $\begin{array}{l}\pi \\
u \\
\sigma\end{array}$ \\
\hline 5. & $>\theta_{2}$ & $\sigma$ & $\begin{array}{l}>\theta_{2} \\
=\theta_{2} \\
<\theta_{2}\end{array}$ & $\begin{array}{l}\sigma \\
u \\
\pi\end{array}$ & $\begin{array}{l}<\theta_{2} \\
<\theta_{2} \\
>\theta_{1}\end{array}$ & $\begin{array}{c}\pi \\
\pi, u^{\pi} \text {, or } \sigma\end{array}$ \\
\hline
\end{tabular}

$\left(\theta_{1}=35^{\circ} 16^{\prime}, \theta_{2}=54^{\circ} 44^{\prime}\right)$

orientation direction, is given by $\mathrm{R}=2 \cot ^{2} \phi$, where $\phi$ is the angle between the transition moment and the orientation direction. ${ }^{3}$ If $\phi=54^{\circ} 44^{\prime}, \mathrm{R}=1$, i.e., the band exhibits no dichroism $(u)$; whereas for $\phi<54^{\circ} 44^{\prime}$ the band exhibits parallel dichroism $(\pi)$, and for $\phi>54^{\circ} 44^{\prime}$ the band exhibits perpendicular dichroism $(\sigma)$.

The various dichroic relationships which are possible can be obtained by examining the angles $\phi_{1}, \phi_{2}$, and $\phi_{3}$ made by the transition moments $\mathbf{M}_{1}, \mathbf{M}_{2}$, and $\mathbf{M}_{3}$, respectively, with the orientation direction. For $0<\phi_{1}<\pi / 2$, the appropriate range for $\phi_{2}$ is $\left(\pi / 2-\phi_{1}\right)$ $<\phi_{2}<\pi / 2$. The angle $\phi_{3}$ is then determined by the condition $\cos ^{2} \phi_{1}+\cos ^{2} \phi_{2}+\cos ^{2} \phi_{3}=1$. The cases which can arise (assuming perfect orientation) are listed in Table I, together with the dichroism of the associated bands. It will be noted that only four combinations are permitted for the dichroism of the three bands : $\sigma \sigma \pi, \pi \pi \sigma, \pi \sigma u$, and wun. The results in Table I are not seriously affected by small deviations from perfect orientation. For example, a simple calculation shows that if all of the transition moments are not oriented at a single angle, $\phi$, but are uniformly distributed in a $20^{\circ}$ angular range about some mean angle, then this mean angle is $54^{\circ} 54^{\prime}$ for $R=1$, compared to $54^{\circ} 44^{\prime}$ for the case of perfect alignment.

These results are especially pertinent to cases where two of the modes associated with a unit exhibit parallel polarization. Although the $\nu_{s}\left(\mathrm{CH}_{2}\right)$ and $\nu_{a}\left(\mathrm{CH}_{2}\right)$ modes are usually perpendicularly polarized, as for example in polyethylene ${ }^{1}$ and polyvinyl alcohol, ${ }^{2}$ in some instances the orientation of this group is such that parallel polarization is observed for both of these modes. This seems to be the case for cellulose $e^{4}$ and polyethylene terephthalate $^{5}$ (see Fig. 1). On the basis of the previously developed results, it would be predicted that in polyethylene terephthalate the $\gamma_{w}\left(\mathrm{CH}_{2}\right)$ mode should be associated with a $\sigma$ band. Therefore, the assignment ${ }^{6}$ of this mode to a $\pi$ band at $1345 \mathrm{~cm}^{-1}$ needs further explanation. A more reasonable assignment of this mode in polyethylene terephthalate is to the $\sigma$ band at 1375 


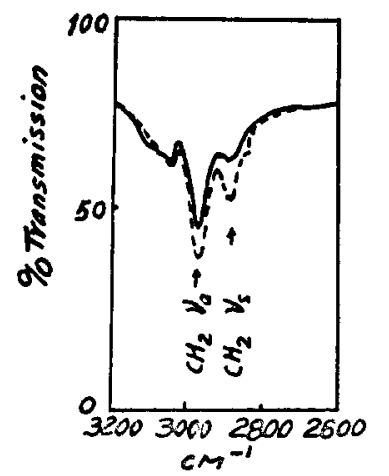

Fig. 1. $\nu_{s}\left(\mathrm{CH}_{2}\right)$ and $v_{a}\left(\mathrm{CH}_{2}\right)$ modes of stretched polyethylene terephthalate. - . - Electric vector parallel to direction of orientation. - Electric vector perpendicular to direction of orientation.

$\mathrm{cm}^{-1}$, which is in agreement with the assignment derived from studies on polyethylene. ${ }^{1}$ Two $\pi$ bands have also been observed for the $\gamma_{w}(\mathrm{OH})$ and $\delta(\mathrm{OH})$ modes of polyvinyl alcohol ${ }^{2}$ (although the situation may be complicated in this case by mixing of modes and interactions between chains ${ }^{2}$ ). We would therefore predict that the $\nu(\mathrm{OH})$ mode should be perpendicularly polarized, and this is in agreement with the observations. ${ }^{2,7}$

\footnotetext{
* Present address : Research and Development Division, American Viscose Corporation, Marcus Hook, Pennsylvania.

${ }^{1}$ Krimm, Liang, and Sutherland, J. Chem. Phys. 25, 549 (1956).

2 Krimm, Liang, and Sutherland, J. Polymer Sci. 22, 227 (1956).

${ }^{3}$ See, for example, R. D. B. Fraser, J. Chem. Phys. 21, 1511 (1953); ibid. 24, 89 (1956); M. Beer, Proc. Roy. Soc. (London) A236, 136 (1956)

${ }^{4}$ M. Tsuboi, J. Polymer Sci. 25, 159 (1957)

SA detailed analysis of the polyethylene terephthalate spectrum will be published soon from this laboratory.

${ }^{6}$ R. G. J. Miller and H. A. Willis, Trans. Faraday Soc. 49, 433 (1953).

7 Glatt, Webber, Seaman, and Ellis, J. Chem. Phys. 18, 413 (1950).
}

\section{Infrared Absorption Bands in $\alpha$-Quartz}

\author{
DARWIN L. WOOD \\ Bell Telephone Laboratories, Murray Hill, New Jersey
} (Received September 13, 1957)

$I^{\mathrm{T}}$ was observed some time ago ${ }^{1}$ that certain infrared absorption bands of crystalline $\alpha$-quartz in the vicinity of $3400 \mathrm{~cm}^{-1}$ do not obey Lambert's law relating thickness to optical density. The absorption coefficients of this group of at least nine bands we find are variable $\left(0.13\right.$ to $\left.6.0 \mathrm{~cm}^{-1}\right)$ from one crystal to another. The origin of the bands must therefore lie in a defect of the lattice which is present in variable concentration in different specimens. The band contours are shown in Fig. 1 for a sample at room temperature and at $77^{\circ} \mathrm{K}$.

Three possible types of defect could be considered in accounting for the bands. (1) Protons may be bound to the lattice to form $\mathrm{OH}$ groups whose stretching vibrations perpendicular to the $c$ axis of the crystal would give both the observed frequency (3200 to $3600 \mathrm{~cm}^{-1}$ ) and dichroism (absorption of the ordinary ray). (2) An oxygen vacancy in the lattice may have around it an electronic system within which a transition of this very low energy may take place. (3) A substitutional or interstitial impurity atom could also account for an electronic transition of this magnitude.

Considering these possibilities we tentatively reject (1) on the basis of two experiments in which the intensities of the bands were altered but presumably only electrons were affected. First the sample was irradiated with about $10^{6} \mathrm{r}$ of $\mathrm{x}$-rays from a tungsten target tube operated at $50 \mathrm{pkv}$ and $50 \mathrm{ma}$ with a $\mathrm{Be}$ window. This caused the intensity of a line at $3600 \mathrm{~cm}^{-1}$ to diminish, while that at $3390 \mathrm{~cm}^{-1}$ increased, and that at 3300 $\mathrm{cm}^{-1}$ remained unchanged. Second, these changes were reversed by heating above $300^{\circ} \mathrm{C}$ for about 10 minutes, restoring the original intensities of the bands. These experiments, though not conclusive suggest an electronic band system rather than a molecular group vibration.

We reject (2) for the following reason. Mitchell and Paige $^{2}$ have suggested that the ultraviolet band at $210 \mathrm{~m} \mu$ in neutron irradiated quart $z$ is associated with oxygen vacancies produced by the irradiation, and that the presence of this band in $x$-irradiated quartz can be attributed to these defects occurring naturally. This band was present in several crystals, but it was not correlated with the presence nor with the changes of the infrared bands as one would expect if both bands originated in oxygen vacancies.

Alternative (3) requires identification of the atom impurity which is causing the bands. Clearly, substitutional aluminum is not involved. Griffiths, Owen, and Ward $^{3}$ have shown that the visible color center of $x$ irradiated and of natural smoky quartz is due to this defect. We find no correlation between the intensity of the visible color center and either the changes in the infrared bands or the original band intensities, and conclude that the two band systems have different origins.

The infrared bands are affected by two types of experiment which change the distribution of interstitial atoms within the lattice. First, heating to $950^{\circ} \mathrm{C}$ decreases the intensity of the infrared bands. At this temperature we find that interstitial aluminum replaces silicon in the lattice since there is an increase in the number of visible $\mathrm{x}$-ray color centers in crystals treated this way. Other interstitial atoms may also be mobile at this temperature, and one can conclude that these are affecting the infrared electronic system.

The second experiment with interstitial atoms involves heating the crystal to $450^{\circ} \mathrm{C}$ for 16 hours in an electric field of about 3000 volts $/ \mathrm{cm}$ parallel to the c-axis. This treatment causes the infrared bands to increase in intensity in some crystals, while others do not respond. We have demonstrated the flow of potassium ions by $\mathrm{x}$-ray fluorescence analysis of the cathode surface, and presume that other alkali cations also move. This result again suggests that interstitial atoms affect the infrared absorption bands. It is interesting to note in passing that heating the crystal in a field will rapidly destroy the visible color centers attributed to 